\title{
Structural Design and Numerical Analysis of Bionic Wheel
}

\author{
Gui-Fen ZHOU ${ }^{1, a}$, Song-Song Ma, ${ }^{2, b}$, Rui ZHANG ${ }^{2, c, *}$ \\ ${ }^{1}$ College of Engineering and Technology, Jilin Agricultural University, Changchun, 130118, China \\ ${ }^{2}$ Key Laboratory of Bionic Engineering, Ministry of Education, Jilin University, Changchun, 130022 , \\ China \\ aguifenzhou@163.com, b604188594@qq.com, zhangrui@jlu.edu.cn \\ ${ }^{*}$ Corresponding author
}

Keywords: Ostrich Foot, Camber Line of Plantar Surface, Bionic Wheel, PFC3D.

\begin{abstract}
Based on the structural characteristic and superior performance of traveling on sand of ostrich foot, explored the special role of the ostrich plantar morphology in the process of traveling on sand. Measured the three-dimensional (3D) point cloud of ostrich plantar surface using 3D laser scanner, and then the 3D irregular surface topography of ostrich foot was acquired using Reverse Engineering (RE) software Geomagic Studio. Extracted camber line of plantar surface of the 3rd toe using software CATIA, carried out optimization and fitting on the characteristic curve, and stretched along width direction of the wheel, then obtained the bionic constitutional unit of bionic wheel traveling on sand, distributed the constitutional unit on the wheel facing evenly. Established the wheel-soil contact model using Discrete element method (DEM) software PFC3D, then performed numerical simulation on the wheel in the sand-striding process. Results show the bionic wheel has excellent anti-subsidence and sand-fixing properties, and effectively improved the traction force and bearing capacity.
\end{abstract}

\section{Introduction}

Due to flowing of sand underneath the wheel, wheel slip sunken as well as greater traction difficult to be provided, seriously affect the trafficability characteristic, even losing the capacity of operating $^{[1-2]}$. The relevant experts and scholars have carried out numerous research on sand-striding wheel, and achieved certain research findings ${ }^{[3-4]}$. However, vehicles trafficability characteristic is improved by means of traditional design methods and technology at present, while break through in the design respect is sparse. Through a lengthy evolution process, ostrich have developed good adaptability to the desert area environment, formed an excellent performance for traveling on sand ${ }^{[5]}$. Based on the sand-striding mechanism of the key parts contacting with sand of animals living in desert environment, adopting technology of Bionics Engineering, combining research of key factors for sand-striding of animals with sand-striding vehicles and desert operating equipment travelling gear will be a break through to the traditional design and research ideas.

African ostrich is the largest bird extant living in the desert, which is a native of Africa and Arabia desert, mainly lives in the open territory and steppe. Although wings have been degraded and unable to fly, the hind limbs possess a steady, durable and high-speed running ability in the sandy environment ${ }^{[6]}$. Foot is the main weight-bearing part, directly contact with the sand and participate in the locomotion, plays a vital role in the performance of sand-striking. In the running and walking process of the ostrich, the foot has excellent sand-binding, anti-subsidence and anti-slipping properties.

The paper took ostrich foot as the biological prototype, according to the structure of plantar surface as well as the sand-striding mechanism, combined with the principle of Bionics Engineering, devised 
a bionic sand-striding wheel with satisfactory trafficability, simulated the interaction relationship between bionic sand-striding wheel and sand by means of DEM, and verified that the bionic sand-striding wheel bears a superior anti-subsidence, higher load-bearing ability and sand-binding properties.

The paper took ostrich foot as the biological prototype, according to the structure of plantar surface as well as the sand-striding mechanism, combined with the principle of Bionics Engineering, devised a bionic sand-striding wheel with satisfactory trafficability, simulated the interaction relationship between bionic sand-striding wheel and sand by means of DEM, and verified that the bionic sand-striding wheel bears a superior anti-subsidence, higher load-bearing ability and sand-binding properties.

\section{Methods}

There are only two toes on the ostrich foot, namely the 3rd toe and the 4th toe, the 3rd toe is larger with big and hard toenail at the anterior part, toe nail of the 4th toe has degenerated and disappeared, as shown in Figure 1. In the locomotion process of ostrich, the 3rd toe mainly contact with sand, plays a role of sand-binding.

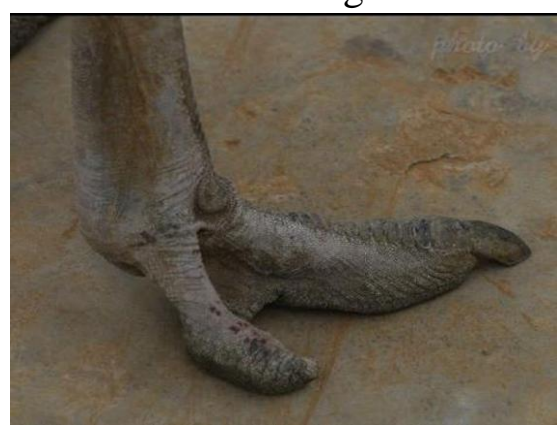

Fig.1 Ostrich Foot Appearance

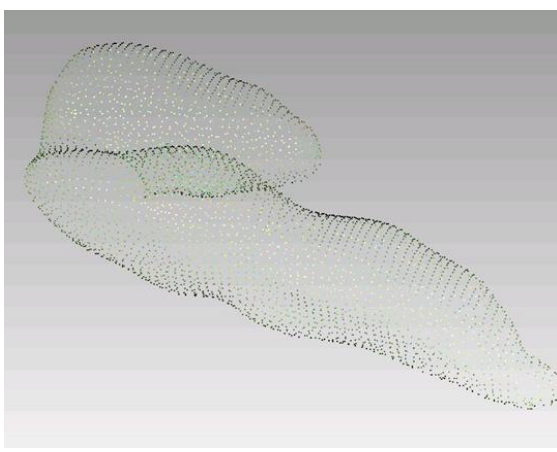

Fig.2 The Original Spatial Point Cloud of Plantar Surface

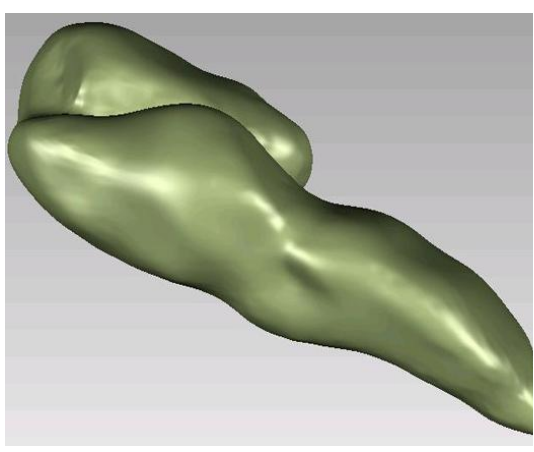

Fig.3 The Original Spatial Point Cloud of Plantar Surface

Adult female ostrich's foot was selected as the research object. Acquired spatial point cloud using non-contact hand-held 3D scanner Rexcan III-0.8M (500 myria pixel, 0.05mm accuracy). Operating principle of the scanner is projects laser onto the object scanned first, then shoot the objects using two cameras, calculated the coordinates of the object's surface using the data obtained. Specimen scanning completed, remove the unnecessary noise points. Fig.2 describes the original spatial point cloud of plantar surface. Based on the principle of reverse engineering, imported the point cloud data of ostrich foot plantar surface into RE software for noise removal, filtering, smoothing, gridding and other processing, and obtained 3D irregular surface of ostrich pelma, which was used for the follow-up characteristic curve extraction of plantar surface, as depicts on Fig.3.

The plantar 3D irregular surface processed above was inported into software CATIA, extracted the effective contour line of ostrich foot plantar 3D irregular surface, namely the ridge line of plantar surface of the 3 rd toe, using engineering drawing projection function. Imported the ridge line of plantar surface of the 3rd toe extracted into sketch module, through optimization and analysis, it is smoothly linked up by seven characteristic curves. Through plotted out by artistic spline line, extracted the curves, and carried out smoothing treatment, the seven circular arcs and their smoothly linking is as depicted on Fig.4 and the Table 1. 


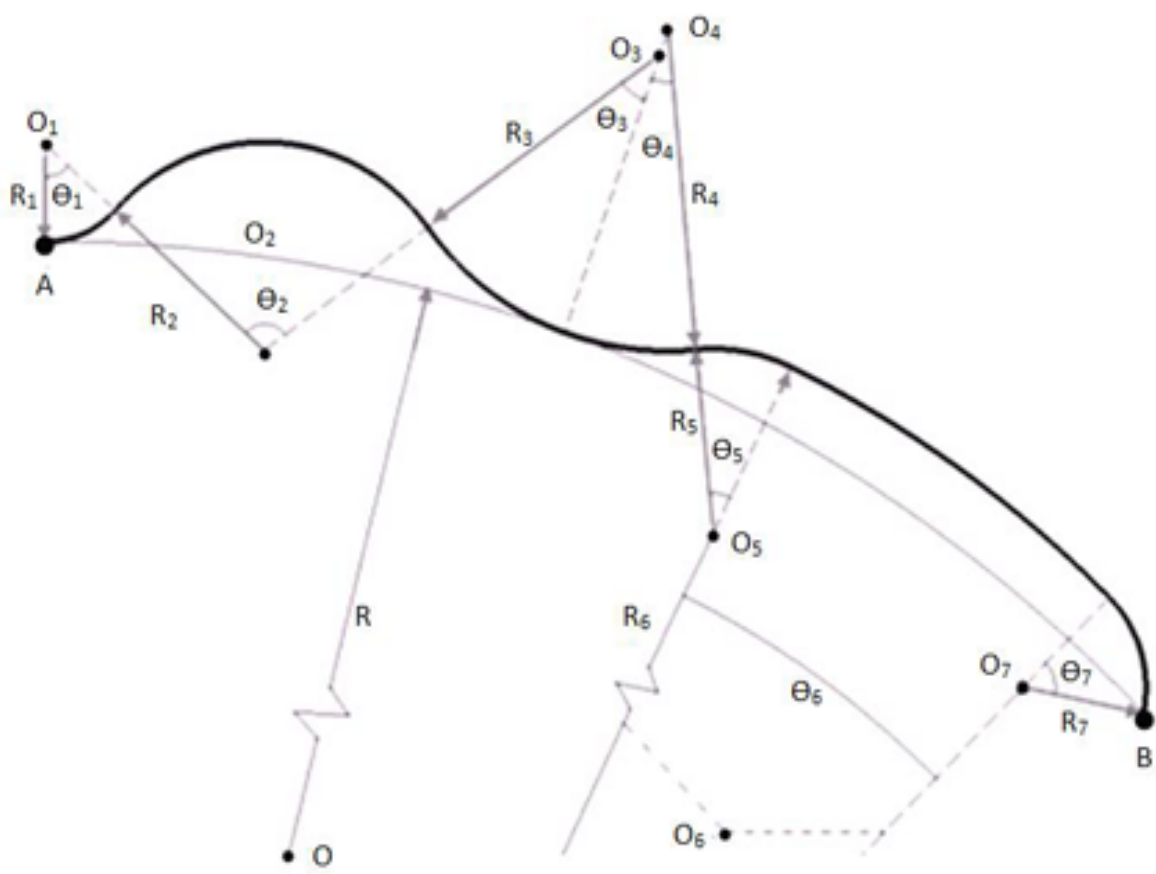

Fig.4 Quantitative Indicator Diagram of the Curve Formed by Characteristic Line

Tab.1 Parameter List of Characteristic Arcs

\begin{tabular}{|c|c|c|c|}
\hline Arc & $\begin{array}{l}\text { Coordinates of } \\
\text { center of circle } \\
(\mathrm{mm})\end{array}$ & $\begin{array}{l}\text { Central } \\
\text { angle } \\
\left({ }^{\circ}\right)\end{array}$ & $\begin{array}{l}\text { Radius } \\
(\mathrm{mm})\end{array}$ \\
\hline Benchmark & $\mathrm{O}=(0,0)$ & & $\mathrm{R}=150.0$ \\
\hline Arc. 1 & $\mathrm{O}_{1}=(0,-159.2)$ & $\theta_{1}=47.47$ & $\mathrm{R}_{1}=9.2$ \\
\hline Arc. 2 & $\mathrm{O}_{2}=(-21.37,-159.60$ & $\theta_{2}=101.65$ & $\mathrm{R}_{2}=19.8$ \\
\hline Arc. 3 & $\mathrm{O}_{3}=(-60.29,-167.69$ & $\theta_{3}=34.40$ & $\mathrm{R}_{3}=28.2$ \\
\hline Arc. 4 & $\mathrm{O}_{4}=(-61.07,-169.86$ & $\theta_{4}=24.79$ & $\mathrm{R}_{4}=30.5$ \\
\hline Arc. 5 & $\mathrm{O}_{5}=(-65.27,-121.94$ & $\theta_{5}=30.80$ & $\mathrm{R}_{5}=17.6$ \\
\hline Arc. 6 & $\mathrm{O}_{6}=(-22.67,-33.97)$ & $\theta_{6}=19.05$ & $\mathrm{R}_{6}=115.3$ \\
\hline Arc. 7 & $\mathrm{O}_{7}=(-95.54,-107.14$ & $\theta_{7}=56.69$ & $\mathrm{R}_{7}=12.1$ \\
\hline
\end{tabular}

Made the lowest point of the plantar characteristic curve in coincidence with the wheel surface of the benchmark wheel, stretched the characteristic line along the width direction of wheel surface, then obtained the bionic constitutional unit of sand-striding wheel face, as shown in Fig.5, and then obtained the bionic constitutional unit of sand-striding wheel face, as shown in Fig.6.

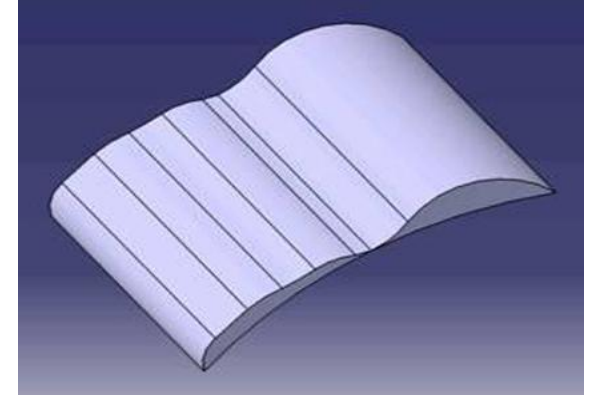

Fig.5 Bionic Constitutional Unit of Sand-striding Wheel Face

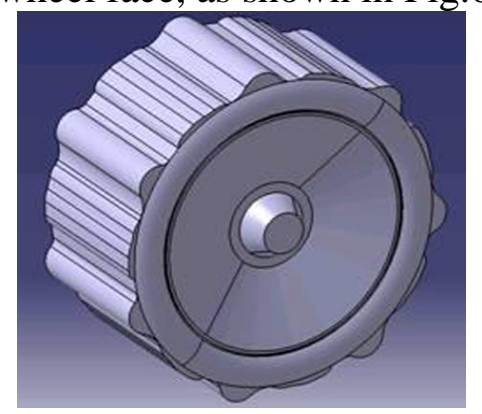

Fig.6 Bionic Wheel Entity 
In order to analyze the performance of bionic sand-striding wheel, we established the desert hiking simulation system of bionic wheel using DEM software PFC3D, analyzed the interaction relationship between wheel facing structure and sand from the perspective of macroscopic and microscopic. Fig.7 shows the initial state of the bionic wheel striking sand. In the discrete element mechanical model of sand, the elastic and nonelastic properties among sand particles were represented by spring and damper. Spring represents for the elasticity of sand particles, damper represents for the non-elasticity of sand particles, the friction between sand particles is represented by a slider with a friction factor, the mechanical contact model $^{[7]}$ is depicted on Fig.8.

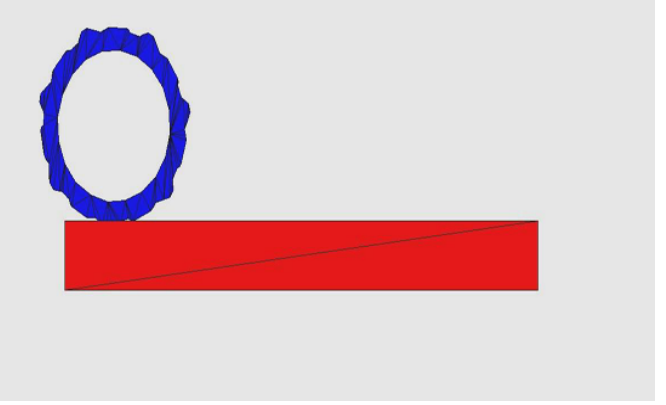

Fig.7 Initial State of the Bionic Wheel Striking Sand

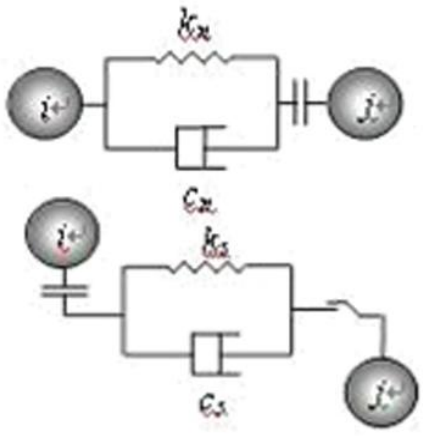

Fig.8 Mechanical Contact Model

Based on physical mechanics parameter dimensional analysis of granular media and simulation analysis of biaxial compression test, the relationship between macro mechanical properties of granular media and parameter of micro contact structure model. Sand on desert Taklimakan was selected as test sample prototype ${ }^{[8]}$, parameters of sand in the simulation process are shown in Table 2 .

Model established, operated the procedure in software PFC 3D, and obtained stress distribution diagram of interaction between wheel and soil. Because in the time point of $T=1.5 \mathrm{~s}$, first half of the bionic wheel is completely touching with sand, sand particles gradually flowed into the fillister area, and the second half begins to touch the sand, the process can perfectly reflect the stress and moving characteristics of the sand underneath the wheel. Therefore, the time point of $\mathrm{T}=1.5 \mathrm{~s}$ was intercepted for the analysis of macro and micro stress field distribution as shown in Fig.9 and Fig.10.

Tab.2 Parameters Set Up in the Discrete Element Simulation

\begin{tabular}{cc}
\hline Variables & Value \\
\hline Particle density (g/m3) & 1.58 \\
Rigidity (N/m) & $1 * 10^{7}$ \\
Particle proportion & 2.6 \\
Particle frictional coefficient & 0.7 \\
Particle radius (mm) & $1.25-1.8$ \\
Friction factor & 0.65 \\
Gravity (m/s2) & 9.8 \\
Tangential critical damping ratio & 0.62 \\
Normal critical damping ratio & 0.84 \\
Time step (s) & $1.5 * 10^{-4}$ \\
Initial porosity factor & 0.7 \\
\hline
\end{tabular}




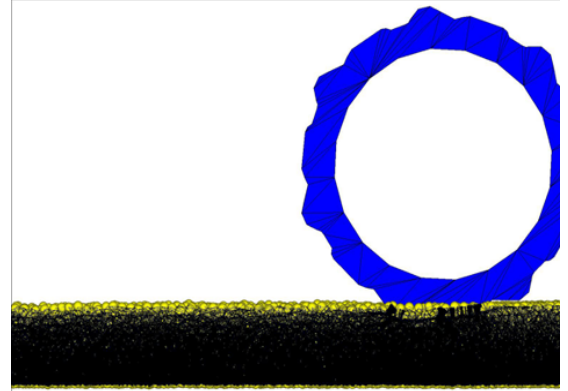

Fig.9 Distribution of Macro Stress Field Underneath The Wheel

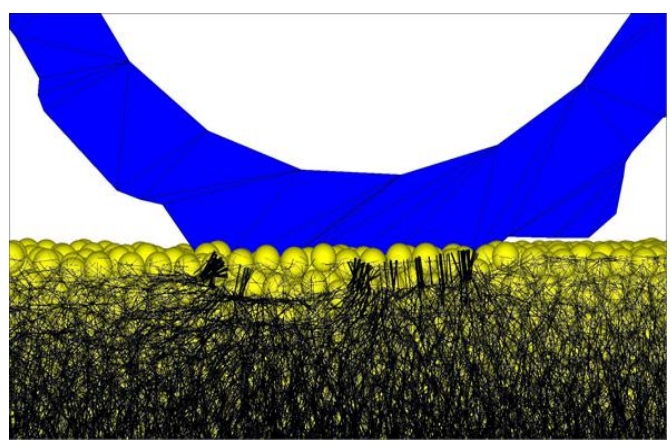

Fig.10 Distribution of Micro Stress Field Underneath the Wheel

\section{Results}

In the whole rolling process of the wheel, the wheel is running steadily, and the slightly stress variation is caused by structural change of wheel. From the microscopic stress field distribution, we came to a conclusion that stress concentration is easily appears in the part where the wheel facing curvature is relatively large. Stress distribution at the anterior part of the wheel structure is homogeneous and located underneath the surface, suggesting that sand is compacted and the fluidity decreased, which is conducive to the enhancement of vehicles travelling on the soft road. In addition, upward thrust of the soil is formed by the constitutional unit of the wheel facing, which can effectively improve the anti-settlement capability and tractive property of the wheel.

\section{Summary}

In this paper, a new type of bionic sand-striding wheel was designed using RE technology, which is based on the structural characteristics and excellent sand-striding property of ostrich foot. Established wheel-soil contact model using DEM software PFC 3D, carried out numerical simulation on the sand-striding process of the bionic sand-striding wheel, analyzed the interaction relationship and stress distribution between bionic sand-striding wheel and soil. We came to the following major conclusions:

(i) Stress of soil underneath the wheel is mainly concentrated in the wheel facing area with bionic characteristic unit, and formed obvious stress concentration in the fillister region imitating the region between heel and palm of ostrich foot, suggesting that the bionic characteristic wheel facing structure is conducive to sand-binding and enhancement of traction.

(ii) Wheelmark formed by the rolling of bionic wheel is distributed uniformly and orderly, and no obvious stress fluctuation underneath the wheel. Stress changing curves of each operation cycle are almost the same, indicating that the bionic sand-striding wheel able to operating smoothly on the soil. (iii) Anterior planar construction of the wheel facing constitutional unit is able to compacting sandy soil, reducing it's fluidity, which is conducive to the enhancement of trafficability characteristic for vehicles travelling on the soft road.

\section{Acknowledgement}

The authors are grateful for the financial support by the National Natural Science Foundation of China (No. 51275199), the Science and Technology Research Project in the 12th Five-Year Period of Education Department of Jilin Province of China (Jijiaokehezi[2015] No. 204) and the Science and Technology Development Planning Project of Jilin Province of China (No. 20140101074JC). 


\section{References}

[1] C. Chen, Development of heavy-duty type desert vehicle is imperative, ZHONGXING QICHE. 3 (1996) 14-15. (in Chinese)

[2] W. Chen, Analysis on Tractive Characteristics of a Wheel on Sands, Tractor \& Farm Transport. 35 (2008) 22-25. (in Chinese)

[3] L.Q. Ren, J. Tong, J.Q. Li, B.C. Chen, Biomechanics of Machinery for Soft Terrain, Transactions of the Chinese Society for Agricultural. 31 (2000) 5-9. (in Chinese)

[4] R. Zhang, M.M. Yang, R.D. Pan, H.B. Liu, G.Y. Zeng, J.Q .Li, Mathematical model establishment of irregular plantar surface of ostrich didactyl foot, Transactions of the Chinese Society of Agricultural Engineering. 31:71-78, 2015. (in Chinese)

[5] N.U. Schaller, D. Kristiaan, V. Rikk, et al. Toe function and dynamic pressure distribution in ostrich locomotion, Journal of Experimental Biology. 214(Pt 7) (2011) 1123-30.

[6] N.U. Schaller, H. Bernd, V. Rikk, et al. The intertarsal joint of the ostrich (Struthio camelus): Anatomical examination and function of passive structures in locomotion.[J]. Journal of Anatomy, 2009, 214(6):830-47.

[7] E. Kasparek, U. Zencker, R. Scheidemann, H. Volzke, K. Muller, Numerical and experimental studies of polyurethane foam under impact loading, COMP MATER SCI. 50 (2011) 1353-1358.

[8] J. Li, X.W Ji., J.D. Zhuang, X.D. Yuan., Experimental study on basic mechanical properties of sand in the Taklimakan Desert of Xinjiang, Automotive Technology. 6(1996) 12-22. (in Chinese) 